\title{
Ferretti-Rajeev Term and Homotopy Theory
}

\author{
Kazuyuki Fujii ${ }^{\star}$ \\ Department of Mathematics, Yokohama City University, Yokohama 236, Japan
}

Received: 15 March 1993/in revised form September 8, 1993

\begin{abstract}
We reduce Ferretti-Rajeev models to the usual sigma models with Chern-Simons terms $(\theta$-terms), and show that whether $\theta$ is quantized or not corresponds to the fact $\pi_{4}\left(G_{j, n}\right) \cong \pi_{3}(U(j))=\mathbb{Z}$ or 0 of the topology in the process of our reduction. We also reconsider the topological invariance of the Chern classes in the language of the field theory.
\end{abstract}

\section{Introduction}

Two dimensional chiral models have widely been studied from both the classical and quantum point of view. See, for example, [16] and its references. But new developments were brought by Witten [15]. Namely he added the so-called Wess-Zumino-Witten term to the kinetic one of chiral models. Then the conformal invariance of the theory was recovered and the boson-fermion correspondence in the quantum level was shown. Unfortunately it is not easy to extend these models in higher dimensions. See, for example, [10].

Next two dimensional nonlinear Grassmann $\sigma$ models have also been studied from both classical and quantum levels and many interesting results have been obtained, see [16]. These models are known as a good toy model for QCD.

Recently Ferretti-Rajeev [3] tried to give a Wess-Zumino-Witten like approach to these models. Namely they proposed new models adding the $2^{\text {nd }}$ Chern class to the kinetic term (of these models). Unlike the Wess-Zumino-Witten models, these ones, of course, do not have the conformal invariance. But these ones are renormalizable in the $1 / N$-expansion, see [3].

They have also rewritten their Grassmann models with chiral fields, and gave current algebra forms in 3-dimensions. In passing to the quantum level, these algebras must be made the abelian extension (not central extension in the case of WZW models), so that it is not easy to treat. See [11], [7].

${ }^{\star}$ Partially supported by the Grant-in-Aid for Scientific Research, No. 04640088 
The aim of this paper is as follows. The target manifold of dynamical variables of F-R models is the Grassmann manifolds $G_{j, n}$. They rewrite F-R models using the unitary group $U(n)$ as stated above. On the other hand we rewrite F-R models using the Stiefel manifold $V_{j, n}$. Then F-R models reduce to the well-known ones "kinetic term $+\theta$ Chern-Simons term" (see, for example, [9]), which is a satisfactory result.

We take interest in the fact whether $\theta$ is quantized or not. Then we show that this corresponds to the fact of the homotopy group $\pi_{4}\left(G_{j, n}\right) \cong \pi_{3}(U(j))=\mathbb{Z}$ or 0 according to $2 \leqq j \leqq n-2$ or $j=1$. Our arguments are more familiar with Witten's ones than that of F-R.

Moreover we consider the case $j=1$ ( $\theta$ is not quantised). Then the theories in the case of $n \geqq 3\left(\mathbb{C} P^{n-1}\right.$-model) are distinct from the case of $n=2\left(\mathbb{C} P^{1}\right.$-model). See [2]. With respect to this point we compare our argument with that of Pak [13] in the appendix.

Our results are summarized as follows: A consistent correspondence between a quantization of $\theta$ and homotopy theory is shown through this paper.

\section{Principal Bundles over Grassmann Manifolds}

Let $\mathbb{C}^{n}$ be the $n$-dimensional complex vector space. For $1 \leqq j \leqq n$ we denote by $M(n, j ; \mathbb{C})$, the $n \times j$-matrices over $\mathbb{C}$. We abbreviate $M(n, n ; \mathbb{C})$ as $M(n ; \mathbb{C})$.

Now we define our manifolds. For $1 \leqq j \leqq n$ we set

$$
\begin{gathered}
V_{j, n}=\left\{Z \in M(n, j ; \mathbb{C}) \mid Z^{\dagger} Z=1_{j}\right\}, \\
G_{j, n}=\left\{P \in M(n ; \mathbb{C}) \mid P^{2}=P, P^{\dagger}=P \text { and } \operatorname{tr} P=j\right\},
\end{gathered}
$$

where $1_{j}$ denotes a unit matrix in $M(j ; \mathbb{C})$.

For

$$
\begin{gathered}
F_{j}=\left(e_{1}, e_{2}, \ldots, e_{j}\right), \\
E_{j}=\left(e_{1}, e_{2}, \ldots, e_{j}, 0, \ldots, 0\right),
\end{gathered}
$$

where $e_{j}$ denotes a $j^{\text {th }}$ unit vector in $\mathbb{C}^{n}$, it is easy to check that

$$
\begin{gathered}
V_{j, n}=\left\{g F_{j} \mid g \in U(n)\right\}, \\
G_{j, n}=\left\{g E_{j} g^{-1} \mid g \in U(n)\right\},
\end{gathered}
$$

where $U(n)$ denotes unitary matrices in $M(n ; \mathbb{C})$. From these facts we have

$$
\begin{gathered}
V_{j, n} \cong U(n) / U(n-j), \\
G_{j, n} \cong U(n) / U(j) \times U(n-j) .
\end{gathered}
$$

We note that $V_{j, n}$ is a real manifold, and $G_{j, n}$ a complex manifold, so that their dimensions are

$$
\begin{gathered}
\operatorname{dim}_{\mathbb{R}} V_{j, n}=2 j(n-j)+j^{2}, \\
\operatorname{dim}_{\mathbb{C}} G_{j, n}=j(n-j) .
\end{gathered}
$$

We also note by (I-8) that

$$
G_{j, n} \cong G_{n-j, n} .
$$


If we define a map

$$
\pi: V_{j, n} \rightarrow G_{j, n}, \quad \pi(Z)=Z Z^{*},
$$

then it is easy to check

$$
\pi(Z)^{2}=\pi(Z), \pi(Z)^{\dagger}=\pi(Z) \text { and } \operatorname{tr} \pi(Z)=j,
$$

so that $\pi(Z)$ defines

$$
\pi: V_{j, n} \rightarrow G_{j, n}
$$

Since

$$
\pi(Z g)=Z g(Z g)^{\dagger}=Z g g^{\dagger} Z^{\dagger}=Z Z^{\dagger}=\pi(Z)
$$

for $g \in U(j)$, this $\pi$ defines a principal $U(j)$-bundle

$$
U(j) \rightarrow V_{j, n} \stackrel{\pi}{\rightarrow} G_{j, n} .
$$

See [5].

At last let us make some preparations from topology. We denote $\pi_{k}(X)$ by the $k^{\text {th }}$ homotopy group of the space $X$.

(a) In the case of $j=1\left(G_{1, n}=\mathbb{C} P^{n-1}\right)$ we have

$$
\begin{aligned}
& \pi_{3}\left(\mathbb{C} P^{n-1}\right)=\left\{\begin{array}{ll}
\mathbb{Z} & \text { if } n=2 \\
0 & \text { if } n \geqq 3
\end{array},\right. \\
& \pi_{4}\left(\mathbb{C} P^{n-1}\right)= \begin{cases}\mathbb{Z}_{2} & \text { if } n=2 \\
0 & \text { if } n \geqq 3\end{cases}
\end{aligned}
$$

(b) In the case of $n-2 \geqq j \geqq 2$ we have

$$
\begin{aligned}
& \pi_{3}\left(G_{j, n}\right)=0, \\
& \pi_{4}\left(G_{j, n}\right)=\mathbb{Z} .
\end{aligned}
$$

We note that for $n \geqq 2$

$$
\begin{gathered}
\pi_{3}(U(n))=\mathbb{Z}, \\
\pi_{4}(U(n))= \begin{cases}\mathbb{Z}_{2} & \text { if } n=2 \\
0 & \text { if } n \geqq 3\end{cases}
\end{gathered}
$$

For the mathematical physicists unfamiliar to these facts, refer to [8] or [14].

\section{Ferretti-Rajeev Models}

Let us make a brief review of the paper of Ferretti-Rajeev [3]. For a dynamical variable

$$
P: S^{3} \rightarrow G_{j, n}
$$


the kinetic term is given by

$$
\begin{aligned}
L_{0} & =\frac{1}{4 f^{2}} \int_{S^{3}} d^{3} x \operatorname{tr} \partial_{\mu} P \partial^{\mu} P \\
& =\frac{1}{4 f^{2}} \int_{S^{3}} \operatorname{tr} d P \wedge * d P,
\end{aligned}
$$

where $f$ is a coupling constant and $*$ is a Hodge dual operator.

By the way since $\pi_{3}\left(G_{j, n}\right)=0$ except for the case $n=2, j=1\left(\mathbb{C} P^{1}\right.$-model $)$ any $P: S^{3} \rightarrow G_{j, n}$ can be extended to

$$
\tilde{P}: B^{4} \rightarrow G_{j, n},\left.\tilde{P}\right|_{S^{3}}=P,
$$

where $B^{4}$ is a 4-dimensional ball whose boundary is $S^{3}$. On the other hand since the cohomology group of $G_{j, n}$ are given by

(a) in the case of $j=1\left(\mathbb{C} P^{n-1}\right.$-model $)$

$$
H^{4}\left(C P^{n-1} ; \mathbb{R}\right)=\mathbb{R} .
$$

(b) in the case of $n-2 \geqq j \geqq 2$

$$
H^{4}\left(G_{j, n} ; \mathbb{R}\right)=\mathbb{R} \oplus \mathbb{R} .
$$

Making use of integrations we can express the action containing $\tilde{P}$ as follows:

$$
\frac{-1}{4 \pi^{2}} \int_{B^{4}} \operatorname{tr}(\tilde{P} d \tilde{P} \wedge d \tilde{P})^{2}
$$

This expression is a bit different from that of F-R [3] apparently. But since $\tilde{P}^{2}=\tilde{P}$, we have easily

$$
\tilde{P} d \tilde{P} d \tilde{P}=d \tilde{P} d \tilde{P} \tilde{P} .
$$

Hereafter we omit the wedge product $\wedge$ of exterior products for simplicity. Therefore we have

$$
\operatorname{tr}(\tilde{P} d \tilde{P} d \tilde{P})^{2}=\operatorname{tr} \tilde{P} d \tilde{P} d \tilde{P} \tilde{P} d \tilde{P} d \tilde{P}=\operatorname{tr} \tilde{P}(d \tilde{P})^{4} .
$$

Under above preparations we introduce a Ferretti-Rajeev term

$$
\begin{aligned}
L_{\mathrm{FR}} \equiv L_{\mathrm{FR}}(P, \tilde{P}) & =\frac{-1}{4 \pi^{2}} \int_{B^{4}} \operatorname{tr}(\tilde{P} d \tilde{P} d \tilde{P})^{2} \\
& =\frac{-1}{4 \pi^{2}} \int_{B^{4}} \operatorname{tr} \tilde{P}(d \tilde{P})^{4}
\end{aligned}
$$

Therefore Ferretti-Rajeev models are given by

$$
L=L_{0}+\theta L_{\mathrm{FR}} \equiv L_{0}(P)+\theta L_{\mathrm{FR}}(P, \tilde{P}) .
$$

This action is defined by a pair $(P, \tilde{P})$ not $P$. But since a lifting $\tilde{P}$ of $P$ is not unique, this action is not single-valued. This fact makes some troubles. But in the Feynman integral approach we only need the fact $e^{\pi i L}$ (not $L$ ) is single-valued. 
For a fixed $P$ in (II-1) let two liftings of $P$ be $\tilde{P}_{+}, \tilde{P}_{-}$, namely

$$
\tilde{P}_{+}, \tilde{P}_{-}: B^{4} \rightarrow G_{j, n},\left.\quad \tilde{P}_{+}\right|_{S^{3}}=\left.\tilde{P}_{-}\right|_{S^{3}}=P .
$$

For the triplet $\left(\hat{P}_{+}, \tilde{P}_{-}, P\right)$ we can construct a map $\hat{P}$

$$
\hat{P}: S^{4} \rightarrow G_{j, n}
$$

as follows:

$$
S^{4}=B_{+}^{4} \cup B_{-}^{4}, B_{+}^{4} \cap B_{-}^{4}=S^{3} \text { and }\left.\hat{P}\right|_{B_{+}^{4}}=\tilde{P}_{+},\left.\hat{P}\right|_{B_{-}^{4}}=\tilde{P}_{-} .
$$

Then it holds

$$
\begin{aligned}
L_{\mathrm{FR}}\left(P, \tilde{P}_{+}\right)-L_{\mathrm{FR}}\left(P, \tilde{P}_{-}\right) & =\frac{-1}{4 \pi^{2}} \int_{B^{4}} \operatorname{tr}\left(\tilde{P}_{+} d \tilde{P}_{+} d \tilde{P}_{+}\right)^{2}-\frac{-1}{4 \pi^{2}} \int_{B^{4}} \operatorname{tr}\left(\tilde{P}_{-} d \tilde{P}_{-} d \tilde{P}_{-}\right)^{2} \\
& =\frac{-1}{4 \pi^{2}} \int_{B^{4} \cup-B^{4}} \operatorname{tr}(\hat{P} d \hat{P} d \hat{P})^{2}=\frac{-1}{4 \pi^{2}} \int_{S^{4}} \operatorname{tr}(\hat{P} d \hat{P} d \hat{P})^{2}
\end{aligned}
$$

Here we took a orientation of $B^{4}$ into consideration. That is, $B^{4}=B_{+}^{4}$ and $-B^{4}=B_{-}^{4}$. For simplicity we set

$$
C_{2}(\hat{P})=\frac{-1}{32 \pi^{2}} \int_{S^{4}} \operatorname{tr}(\hat{P} d \hat{P} d \hat{P})^{2} .
$$

This is a well-known Chern class.

By the way since $\pi_{4}\left(\mathbb{C} P^{n-1}\right)=0$ for $n \geqq 3$ and $\pi_{4}\left(G_{j, n}\right)=\mathbb{Z}$ for $n-2 \geqq j \geqq 2$ by (I-16), (I-18), we have

$$
\begin{array}{ll}
C_{2}(\hat{P})=0 & \text { for } \quad C P^{n-1}, \\
C_{2}(\hat{P}) \in \mathbb{Z} & \text { for } \quad G_{j, n} .
\end{array}
$$

Therefore

$$
\begin{aligned}
& e^{i\left\{L_{0}(P)+\theta L_{\mathrm{FR}}\left(P, \tilde{P}_{+}\right)\right\}}=e^{i\left\{L_{0}(P)+\theta L_{\mathrm{FR}}\left(P, \tilde{P}_{-}\right)\right\}} \\
& \Leftrightarrow e^{i \theta\left\{L_{\mathrm{FR}}\left(P, \tilde{P}_{+}\right)-L_{\mathrm{FR}}\left(P, \tilde{P}_{-}\right)\right\}}=1 \\
& \Leftrightarrow e^{i \theta 8 \mathrm{C}_{2}(\hat{P})}=1
\end{aligned}
$$

Here we must take two cases into consideration.

(a) $j=1\left(C P^{n-1}\right.$-model) and $n \geqq 3$. In this case since $C_{2}(\hat{P})=0$ (II-20) holds automatically. Therefore $\theta$ takes any values, so that $\theta$ is not quantized.

(b) $n-2 \geqq j \geqq 2\left(G_{j, n}\right.$-model $)$. In this case $C_{2}(\hat{P}) \in \mathbb{Z}$. But since $C_{2}(\hat{P})$ takes any integers by changing pair $\left(\tilde{P}_{+}, \tilde{P}_{-}\right)$of $P$, so that (II-20) holds if

$$
\theta=\frac{\pi}{4} n, \quad n \in \mathbb{Z}
$$

Namely $\theta$ is not admitted to take any values, so $\theta$ is quantized.

We note that we cannot take a Wess-Zumino-Witten like approach to the $\mathbb{C} P^{1}$-model because $\pi_{3}\left(\mathbb{C} P^{1}\right)=\pi_{3}\left(S^{2}\right)=\mathbb{Z}$. 


\section{Reduction of Ferretti-Rajeev Models}

The action form (II-12) of F-R models is unbalance because it contains both $P$ and its lifting $\tilde{P}$. To remove it we change a dynamical variable $P$. Hereafter we take an another route from F-R.

For each point $p t \in B^{4}, \tilde{P}(p t)$ can be factorized as follows

$$
\tilde{P}(p t)=\tilde{Z}(p t) \tilde{Z}(p t)^{\dagger} .
$$

by (I-12). By the way since $B^{4}$ is contractible, $\tilde{P}$ can be factorized on the whole $B^{4}$,

$$
\tilde{P}=\tilde{Z} \tilde{Z}^{\dagger}, \quad \tilde{Z}: B^{4} \rightarrow V_{j, n} .
$$

Therefore we have

$$
P=Z Z^{\dagger}, \quad Z=\left.\tilde{Z}\right|_{S^{3}}: S^{3} \rightarrow V_{j, n},
$$

since $P=\left.\tilde{P}\right|_{S^{3}}$. Hereafter we use $(Z, \tilde{Z})$ instead of $(P, \tilde{P})$.

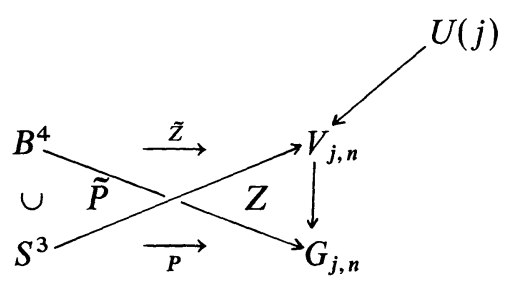

For simplicity we now set

$$
\tilde{A}=\tilde{Z}^{\dagger} d \tilde{Z}, \quad A=Z^{\dagger} d Z .
$$

Then it is easy to check that

$$
\operatorname{tr}(\tilde{P} d \tilde{P} d \tilde{P})^{2}=\operatorname{tr}\left(d \tilde{A}+\tilde{A}^{2}\right)^{2}=d \operatorname{tr}\left(\tilde{A} d \tilde{A}+\frac{2}{3} \tilde{A}^{3}\right) .
$$

See [1], [10]. The right-hand side of (III-4) is a well-known Chern-Simons form. Therefore F-R action can be rewritten as follows:

$$
\begin{aligned}
& \frac{1}{4 f^{2}} \int_{S^{3}} \operatorname{tr} d P \wedge * d P+\frac{-\theta}{4 \pi^{2}} \int_{B^{4}} \operatorname{tr}(\tilde{P} d \tilde{P} d \tilde{P})^{2} \\
& =\frac{1}{4 f^{2}} \int_{S^{3}} \operatorname{tr} d P \wedge * d P+\frac{-\theta}{4 \pi^{2}} \int_{B^{4}} d \operatorname{tr}\left(\tilde{A} d \tilde{A}+\frac{2}{3} \tilde{A}^{3}\right), \\
& =\frac{1}{4 f^{2}} \int_{S^{3}} \operatorname{tr} d P \wedge * d P+\frac{-\theta}{4 \pi^{2}} \int_{S^{3}} \operatorname{tr}\left(A d A+\frac{2}{3} A^{3}\right),
\end{aligned}
$$

or, in the form using $Z$,

$$
\begin{aligned}
= & \frac{1}{2 f^{2}} \int_{S^{3}} \operatorname{tr}\left\{d Z^{\dagger} * d Z+Z^{\dagger} d Z *\left(Z^{\dagger} d Z\right)\right\} \\
& +\frac{-\theta}{4 \pi^{2}} \int_{S^{3}} \operatorname{tr}\left\{Z^{\dagger} d Z d\left(Z^{\dagger} d Z\right)+\frac{2}{3}\left(Z^{\dagger} d Z\right)^{3}\right\}
\end{aligned}
$$


or, in the usual form,

$$
\begin{aligned}
= & \frac{1}{2 f^{2}} \int_{S^{3}} d^{3} x \operatorname{tr}\left(\partial_{\mu} Z^{\dagger} \partial^{\mu} Z+Z^{\dagger} \partial_{\mu} Z Z^{\dagger} \partial^{\mu} Z\right) \\
& +\frac{-\theta}{4 \pi^{2}} \int_{S^{3}} d^{3} x \varepsilon^{\mu \nu \lambda} \operatorname{tr}\left\{Z^{\dagger} \partial_{\mu} Z \partial_{\nu}\left(Z^{\dagger} \partial_{\lambda} Z\right)+\frac{2}{3} Z^{\dagger} \partial_{\mu} Z Z^{\dagger} \partial_{\nu} Z Z^{\dagger} \partial_{\lambda} Z\right\}
\end{aligned}
$$

Now removing the boundary condition of $Z$, we take $Z$ as

$$
Z: \mathbb{R} \times S^{2} \rightarrow V_{j, n}
$$

Then our model is given by

$$
L=L_{0}+\theta L_{\mathrm{CS}} \equiv L_{0}(Z)+\theta L_{\mathrm{CS}}(Z),
$$

where

$$
\begin{aligned}
L_{0}= & \int_{\mathbb{R} \times S^{2}} d^{3} x \mathfrak{I}_{0} \\
= & \frac{1}{2 f^{2}} \int_{\mathbb{R} \times S^{2}} d^{3} x \operatorname{tr}\left(\partial_{\mu} Z^{\dagger} \partial^{\mu} Z+Z^{\dagger} \partial_{\mu} Z Z^{\dagger} \partial^{\mu} Z\right) \\
& L_{\mathrm{CS}}=\int_{\mathbb{R} \times S^{2}} d^{3} x \mathfrak{L}_{\mathrm{CS}} \\
= & \frac{-\theta}{4 \pi^{2}} \int_{\mathbb{R} \times S^{2}} d^{3} x \varepsilon^{\mu \nu \lambda} \operatorname{tr}\left\{Z^{\dagger} \partial_{\mu} Z \partial_{v}\left(Z^{\dagger} \partial_{\lambda} Z\right)+\frac{2}{3} Z^{\dagger} \partial_{\mu} Z Z^{\dagger} \partial_{\nu} Z Z^{\dagger} \partial_{\lambda} Z\right\}
\end{aligned}
$$

For the abelian case $(j=1)$

$$
\begin{aligned}
L_{0} & =\frac{1}{2 f^{2}} \int_{\mathbb{R} \times S^{2}} d^{3} x\left(\partial_{\mu} Z^{\dagger} \partial^{\mu} Z+Z^{\dagger} \partial_{\mu} Z Z^{\dagger} \partial^{\mu} Z\right), \\
L_{\mathrm{CS}} & =\frac{-\theta}{4 \pi^{2}} \int_{\mathbb{R} \times S^{2}} d^{3} x \varepsilon^{\mu \nu \lambda} Z^{\dagger} \partial_{\mu} Z \partial_{\nu}\left(Z^{\dagger} \partial_{\lambda} Z\right) .
\end{aligned}
$$

We introduced unnecessary degrees of freedom to represent the F-R action in a local form. Namely we used $Z$ instead of a pair $(P, \widetilde{P})$. Therefore we must investigate influences which unnecessary degrees of freedom act our model.

For a transformation

$$
Z \rightarrow Z g, \quad g: \mathbb{R} \times S^{2} \rightarrow U(j),
$$

$Z^{\dagger} d Z$ transforms

$$
Z^{\dagger} d Z \rightarrow g^{-1} Z^{\dagger} d Z g+g^{-1} d g
$$

so that we have

$$
\begin{aligned}
\mathfrak{L}_{0} \rightarrow & \mathfrak{L}_{0} \\
\mathfrak{L}_{\mathrm{CS}} \rightarrow & \mathfrak{L}_{\mathrm{CS}}-\frac{1}{4 \pi^{2}} \varepsilon^{\mu \nu \lambda} \partial_{\mu} \operatorname{tr}\left(Z^{\dagger} \partial_{\nu} Z \partial_{\lambda} g g^{-1}\right) \\
& \quad+\frac{1}{12 \pi^{2}} \varepsilon^{\mu \nu \lambda} \operatorname{tr}\left(g^{-1} \partial_{\mu} g g^{-1} \partial_{\nu} g g^{-1} \partial_{\lambda} g\right)
\end{aligned}
$$


after some calculations. See [9]. Namely under the transformation $Z \rightarrow Z g, \mathfrak{L}_{0}$ is invariant and $\mathfrak{L}_{\mathrm{CS}}$ changes only by total divergent term + topological term. Therefore our action (III-9) is not invariant under the above transformation. But in the Feynman integral approach we need only that $e^{4 i L(Z)}$ (not $L(Z)$ ) is invariant.

Here for

$$
g: S^{3} \rightarrow U(j)
$$

(we impose the boundary condition on $g$ ) we set

$$
n(g)=\frac{1}{24 \pi^{2}} \int_{S^{3}} d^{3} x \varepsilon^{\mu \nu \lambda} \operatorname{tr}\left(g^{-1} \partial_{\mu} g g^{-1} \partial_{\nu} g g^{-1} \partial_{\lambda} g\right)
$$

Since $\pi_{3}(U(1))=0$ and $\pi_{3}(U(j))=\pi_{3}(S U(j))=\mathbb{Z}$ for $n \geqq 2$ by (I-19), we have

$$
\begin{array}{ll}
n(g)=0 & \text { for } U(1), \\
n(g) \in \mathbb{Z} & \text { for } U(j)(j \geqq 2) .
\end{array}
$$

Therefore

$$
\begin{aligned}
& e^{4 i\left\{L_{0}(Z)+\theta L_{\mathrm{cs}}(Z)\right\}}=e^{4 i\left\{L_{0}(Z g)+\theta L_{\mathrm{cs}}(Z g)\right\}} \\
& \quad \Leftrightarrow e^{4 i \theta\left\{L_{\mathrm{cs}}(Z g)-L_{\mathrm{cs}}(Z)\right\}}=1 \\
& \Leftrightarrow e^{8 i \theta n(g)}=1
\end{aligned}
$$

Here we must take two cases into consideration.

(a) $j=1(U(1)$-case) and $n \geqq 3$. In this case since $n(g)=0($ III-19) holds automatically. Therefore $\theta$ is not quantized.

(b) $n-2 \geqq j \geqq 2(U(j)$-case). In this case (III-1) must hold for any $n(g) \in \mathbb{Z}$, so that $\theta$ must take

$$
\theta=\frac{\pi}{4} n, \quad n \in \mathbb{Z}
$$

Namely $\theta$ is quantized.

We conclude that a quantization of $\theta$ in F-R models and in our models corresponds to the fact $\pi_{4}\left(G_{j, n}\right) \cong \pi_{3}(U(j))=\mathbb{Z}$ or 0 of the homotopy theory in a perfect manner.

\section{Abelian Chern-Simons Terms}

Let us recall the homotopy groups of $\mathbb{C} P^{n-1}$ once more.

$$
\begin{aligned}
& \pi_{3}\left(\mathbb{C} P^{n-1}\right)= \begin{cases}\mathbb{Z} & \text { if } n=2 \\
0 & \text { if } n \geqq 3,\end{cases} \\
& \pi_{4}\left(\mathbb{C} P^{n-1}\right)= \begin{cases}\mathbb{Z}_{2} & \text { if } n=2 \\
0 & \text { if } n \geqq 3 .\end{cases}
\end{aligned}
$$

Therefore for $\mathbb{C} P^{n-1}$-model $(n \geqq 3), \theta$ is not quantized as discussed in the previous sections. But for $\mathbb{C} P^{1}$-model, our discussion is not applied since $\pi_{3}\left(\mathbb{C} P^{1}\right)=\pi_{3}\left(S^{2}\right)=\mathbb{Z}$. 
We cannot take a Wess-Zumino-Witten like approach to the $\mathbb{C} P^{1}$-model. But, interesting sufficiently, we can consider the action (III-9) with (III-10a) and (III-11b) even in this case. Namely we can add a Hopf term (III-11b) to the kinetic term (III-10a) by hand. In fact such a model has been treated in [2]. But the problems which we treat in this paper and is treated in [2] are completely different.

At any rate we consider the abelian Chern-Simons term

$$
\varepsilon^{\mu v \lambda} Z^{\dagger} \partial_{\mu} Z \partial_{v}\left(Z^{\dagger} \partial_{\lambda} Z\right) \text {. }
$$

Here we omitted the coefficient $\frac{-\theta}{4 \pi^{2}}$ for simplicity. From this term can we understand the difference in (IV-3) between $n=2$ and $n \geqq 3$ ? Let us investigate this point. For that purpose we write $Z$ using a local coordinate.

A parametrization of $Z$ which we use is

$$
Z(\alpha, z)=\left[\begin{array}{c}
1+i \alpha-|z|^{2} \\
2 z
\end{array}\right]\left(1-i \alpha+|z|^{2}\right)^{-1}
$$

where $\alpha \in \mathbb{R}$ and $z=\left(z_{1}, z_{2}, \ldots, z_{n-1}\right)^{t} \in \mathbb{C}^{n-1}$, and $|z|^{2}=z^{\dagger} z . Z(\alpha, z)$ above is also rewritten as

$$
Z(\alpha, z)=\left[\begin{array}{l}
\frac{2}{1-i \alpha+|z|^{2}}-1 \\
\frac{2 z}{1-i \alpha+|z|^{2}}
\end{array}\right] .
$$

Next taking $\alpha$ and $z$ as

$$
\alpha=\alpha\left(x_{0}, x_{1}, x_{2}\right), \quad z_{j}=z_{j}\left(x_{0}, x_{1}, x_{2}\right)
$$

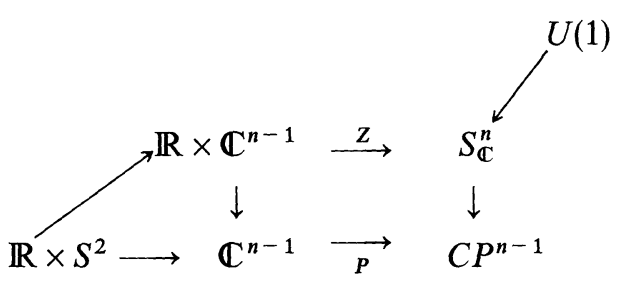

We compute (IV-3). The result is as follows.

If we set

$$
|F|^{2}=\left(1+|z|^{2}\right)^{2}+\alpha^{2}
$$

then we have

$$
(I V-3)=\frac{8}{|F|^{4}} \varepsilon^{\mu v \lambda}\left\{i \partial_{\mu} \alpha-\left(\partial_{\mu} z^{\dagger} z-z^{\dagger} \partial_{\mu} z\right)\right\} \partial_{v} z^{\dagger} \partial_{\lambda} z .
$$

Now we investigate the case $n=2$ in details. From (IV-7) we have

$$
(I V-3)=\frac{8 i}{|F|^{4}} \varepsilon^{\mu \nu \lambda} \partial_{\mu} \alpha \partial_{v} \bar{z} \partial_{\lambda} z
$$


This term cannot be written as a total divergent form. Refer to Appendix. Up to this time we cannot understand the difference between $n=2$ and $n \geqq 3$ apparently. Next we take a variational approach. Namely

$$
\begin{aligned}
& \alpha \rightarrow \alpha+\delta \alpha, \\
& z \rightarrow z+\delta z ; \quad \bar{z} \rightarrow \bar{z}+\delta \bar{z} .
\end{aligned}
$$

Then we have after some calculations

$$
\begin{aligned}
\delta\left(\frac{8 i}{|F|^{4}} \varepsilon^{\mu \nu \lambda} \partial_{\mu} \alpha \partial_{v} \bar{z} \partial_{\lambda} z\right)= & \varepsilon^{\mu \nu \lambda}\left\{\partial_{\mu}\left(\frac{8 i}{|F|^{4}} \delta \alpha \partial_{v} \bar{z} \partial_{\lambda} z\right)\right. \\
& +\partial_{v}\left(\frac{8 i}{|F|^{4}} \partial_{\mu} \alpha \delta \bar{z} \partial_{\lambda} z\right) \\
& \left.+\partial_{\lambda}\left(\frac{8 i}{|F|^{4}} \partial_{\mu} \alpha \partial_{v} \bar{z} \delta z\right)\right\} .
\end{aligned}
$$

This is just a total divergent form. For $n \geqq 3$

$$
\delta\left(\frac{8}{|F|^{4}} \varepsilon^{\mu \nu \lambda}\left\{i \partial_{\mu} \alpha-\left(\partial_{\mu} z^{\dagger} z-z^{\dagger} \partial_{\mu} z\right)\right\} \partial_{v} z^{\dagger} \partial_{\lambda} z\right)
$$

is not rewritten as a total divergent form. It is this point that the difference between $\pi_{3}\left(\mathbb{C} P^{1}\right)=\pi_{3}\left(S^{2}\right)=\mathbb{Z}$ and $\pi_{3}\left(\mathbb{C} P^{n-1}\right)=0(n \geqq 3)$ appears.

In last we compute the Hopf index in the case of $n=2$. By (IV-8)

$$
\frac{-1}{4 \pi^{2}} \int d^{3} x \varepsilon^{\mu \nu \lambda} Z^{\dagger} \partial_{\mu} Z \partial_{\nu}\left(Z^{\dagger} \partial_{\lambda} Z\right)=\frac{-2 i}{\pi^{2}} \int d^{3} x \varepsilon^{\mu \nu \lambda} \frac{\partial_{\mu} \alpha \partial_{\nu} \bar{z} \partial_{\lambda} z}{|F|^{4}} .
$$

Here we choose

$$
\alpha=x_{0}, \quad z=x_{1}+i x_{2} .
$$

Then since

$$
\begin{gathered}
|F|^{2}=\left(1+|z|^{2}\right)^{2}+x_{0}^{2}=\left(1+x_{1}^{2}+x_{2}^{2}\right)^{2}+x_{0}^{2}, \\
\varepsilon^{\mu \nu \lambda} \partial_{\mu} \alpha \partial_{\nu} \bar{z} \partial_{\lambda} z=2 i,
\end{gathered}
$$

we have

$$
\begin{aligned}
(I V-12) & =\frac{4}{\pi^{2}} \int d x_{0} \int d^{2} x \frac{1}{\left\{\left(1+x_{1}^{2}+x_{2}^{2}\right)^{2}+x_{0}^{2}\right\}^{2}} \\
& =\frac{4}{\pi^{2}} \int d^{2} x \frac{\pi / 2}{\left(1+x_{1}^{2}+x_{2}^{2}\right)^{3}} \\
& =\frac{2}{\pi} \times 2 \pi \int_{0}^{\infty} d r \frac{r}{\left(1+r^{2}\right)^{3}}=4 \times \frac{1}{4}=1,
\end{aligned}
$$


where in the process of our calculations we used the fact

$$
\int d \alpha \frac{1}{\left(\alpha^{2}+A^{2}\right)^{2}}=\frac{\pi}{2 A^{3}} \text {. }
$$

Our choice (IV-5) with (IV-13) gives a Hopf index 1.

We note that our discussion of this section is a bit different from that of Pak [13]. We will argue our differences in the appendix.

\section{Topological Invariance of the Chern Classes}

Let us recall the homotopy groups of $G_{j, n}$ once more,

$$
\begin{aligned}
& \pi_{2}\left(G_{j, n}\right)=\mathbb{Z} \text { for } n-1 \geqq j \geqq 1, \\
& \pi_{4}\left(G_{j, n}\right)= \begin{cases}\mathbb{Z} & \text { if } n-2 \geqq j \geqq 2 \\
0 & \text { if } j=1 .\end{cases}
\end{aligned}
$$

These are expressed as Chern forms. For $P: S^{2 k} \rightarrow G_{j, n}$, the $k^{\text {th }}$ Chern class is given by

$$
C_{k}(P)=\frac{1}{2^{k} k !(2 \pi i)^{k}} \int_{S^{2 k}} \operatorname{tr}(P d P d P)^{k},
$$

see [6]. In particular when $k=1$ and $k=2$, we have

$$
\begin{aligned}
& C_{1}(P)=\frac{-i}{4 \pi} \int_{S^{2}} \operatorname{tr} P d P d P, \\
& C_{2}(P)=\frac{-1}{32 \pi^{2}} \int_{S^{4}} \operatorname{tr}(P d P d P)^{2} .
\end{aligned}
$$

Chern classes are well-known as a topological invariant, see [12]. For $P, Q: S^{2 k} \rightarrow G_{j, n}$, which are homotopic $(P \simeq Q)$, then we have

$$
C_{k}(P)=C_{k}(Q) .
$$

But the proof is not so easy. In this section we show the spirit of the proof (of course our proof is not complete) in the case where $k=1$ and $k=2$.

For

$$
U_{i}: S^{2 k} \rightarrow \mathfrak{g}(U(n)),
$$

where $\mathrm{g}(U(n))$ is the Lie algebra of $U(n), e^{U_{i}} P e^{-U_{i}}$ is contained in $\operatorname{Map}\left(S^{2 k}, G_{j, n}\right)$ and homotopic to $P$. Then the connected component in $\operatorname{Map}\left(S^{2 k}, G_{j, n}\right)$ containing $P$ is generated by

$$
Q=e^{U_{i}} e^{U_{i-1}} \ldots e^{U_{1}} P e^{-U_{1}} \ldots e^{-U_{i-1}} e^{-U_{i}}
$$

for appropriate $i$. Therefore if we can show that

$$
C_{k}\left(e^{U} P e^{-U}\right)=C_{k}(P) \text {. }
$$

for $U: S^{2 k} \rightarrow \mathfrak{g}(U(n))$, then we have $C_{k}(Q)=C_{k}(P)$ by inductive arguments. 
For simplicity we set $A=e^{U}$. Remarking that $\operatorname{tr} P d P d P=\operatorname{tr} P d P d P P$ we investigate how two form PdPdPP behaves under the transformation

After short calculations

$$
P \rightarrow A P A^{-1} \text {. }
$$

$$
d\left(A P A^{-1}\right)=A\left(d P+\left[A^{-1} d A, P\right]\right) A^{-1},
$$

so that we have

$$
\begin{aligned}
& A P A^{-1} d\left(A P A^{-1}\right) d\left(A P A^{-1}\right) \\
& \quad=A P\left(d P+\left[A^{-1} d A, P\right]\right)\left(d P+\left[A^{-1} d A, P\right]\right) P A^{-1} .
\end{aligned}
$$

This is a fundamental form of our calculation. Using this we calculate (V-3) in the case where $k=1$ and $k=2$. The result is as follows.

$$
\begin{aligned}
\operatorname{tr}\left\{A P A^{-1} d\left(A P A^{-1}\right) d\left(A P A^{-1}\right)\right\}= & \operatorname{tr} P d P d P+d \operatorname{tr} A^{-1} d A P, \\
\operatorname{tr}\left\{A P A^{-1} d\left(A P A^{-1}\right) d\left(A P A^{-1}\right)\right\}^{2}= & \operatorname{tr}(P d P d P)^{2}+d\left\{A^{-1} d A P d\left(A^{-1} d A P\right)\right. \\
& +\frac{2}{3}\left(A^{-1} d A P\right)^{3}+2 d P d P A^{-1} d A P \\
& \left.+2 d P A^{-1} d A P A^{-1} d A P\right\} .
\end{aligned}
$$

We note that the total divergent term (V-14) contains the Chern-Simons terms

$$
X d X+\frac{2}{3} X^{3}
$$

with $X=A^{-1} d A P$. We have (V-9) from (V-13), (V-14).

Unfortunately we cannot give an explicit form to (V-3) in the case where $n \geqq 3$. We leave it to the readers as an open question.

\section{Conclusion}

We show the difference between F-R method and our method once more.

Let us consider a dynamical variable

$$
\tilde{P}: B^{4} \rightarrow G_{j, n} .
$$

(i) F-R method. They decompose $\tilde{P}$ into

$$
\tilde{P}=\tilde{g} E_{j} \tilde{g}^{-1}, \quad \tilde{g}: B^{4} \rightarrow U(n),
$$

and rewrite the action (II-12) using $\tilde{g}$ and $g=\left.\tilde{g}\right|_{S^{3}}$. The whole diagram is as follows:

$$
U(j) \times U(n-j)
$$

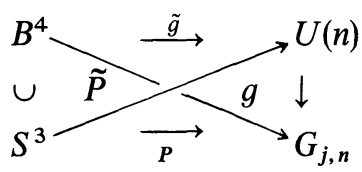


(ii) Our Method. We decompose $\tilde{P}$ into

$$
\tilde{P}=\tilde{Z} \tilde{Z}^{\dagger}, \quad \tilde{Z}: B^{4} \rightarrow V_{j, n},
$$

and rewrite the action (II-12) using $\tilde{Z}$ and $Z=\left.\tilde{Z}\right|_{S^{3}}$. The whole diagram is as follows:

$$
U(j)
$$

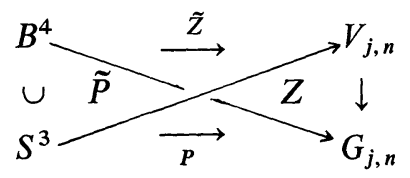

Some remarks are in order.

We have not given a geometric quantization of our model (see Pak [13] for $\mathbb{C} P^{n-1}$-model). The bose-fermi correspondence may exist in our model as suggested in [3]. What is an explicit form? We must also perform a $1 / N$-expansion in the Feynman integral of our model, see [4].

We will investigate these points in future works.

\section{Appendix}

In [13] Pak calculated the abelian Chern-Simons term (the generalized Hopf term in the language of [13])

$$
\varepsilon^{\mu \nu \lambda} Z^{\dagger} \partial_{\mu} Z \partial_{v}\left(Z^{\dagger} \partial_{\lambda} Z\right)
$$

to clarify the difference in the case where $n=2$ and $n \geqq 3$. For that purpose he also used a local coordinate different from ours, see Sect. IV. To clarify the difference in between our method and his one let us make a short review of [13]

His parametrization of $Z$ is

$$
Z=\frac{1}{\sqrt{1+|z|^{2}}}\left[\begin{array}{l}
1 \\
z
\end{array}\right] e^{i \varphi},
$$

where $z=\left(z_{1}, z_{2}, \ldots, z_{n-1}\right)^{t} \in \mathbb{C}^{n-1}$ and $|z|^{2}=z^{\dagger} z, \quad$ and $z_{j}=z_{j}\left(x_{0}, x_{1}, x_{2}\right)$, $\varphi=\varphi\left(x_{0}, x_{1}, x_{2}\right)$,
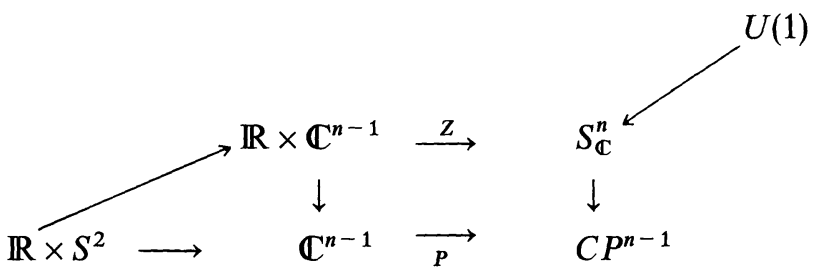

In the strict sense this $Z$ is not a local coordinate of $S_{\mathbb{C}}^{n}$. In this expression each $z_{j}$ and $\varphi$ does not mix. Compare (b) with (IV-5). 
Using this $Z$ let us calculate (a). The result is as follows:

$$
\begin{aligned}
(a)= & \varepsilon^{\mu \nu \lambda} \partial_{\mu}\left\{i \varphi\left(\frac{\partial_{\nu} z^{\dagger} \partial_{\lambda} z}{1+|z|^{2}}+\frac{z^{\dagger} \partial_{\nu} z \partial_{\lambda} z^{\dagger} z}{\left(1+|z|^{2}\right)^{2}}\right)\right\} \\
& +\frac{1}{2} \varepsilon^{\mu \nu \lambda}\left(z^{\dagger} \partial_{\mu} z-\partial_{\mu} z^{\dagger} z\right) \partial_{\nu} z^{\dagger} \partial_{\lambda} z
\end{aligned}
$$

When $n=2\left(\mathbb{C} P^{1}\right.$-model $)$, the last term in (c) drops and (a) reduces to

$$
(a)=\varepsilon^{\mu \nu \lambda} \partial_{\mu}\left\{i \varphi \frac{\partial_{v} \bar{z} \partial_{\lambda} z}{\left(1+|z|^{2}\right)^{2}}\right\}
$$

after a short calculation. That is, the abelian Chern-Simons term (a Hopf term) becomes a total divergent form. When $n \geqq 3$ this term does not become a total divergent form due to the last term in (c).

Note added in proof : In Sect. I (or Sect. IV) we defined two principal bundles over the Grassmann manifold $G_{j, n}$. Here we shall unite two diagrams of principal bundles into one diagram as follows.

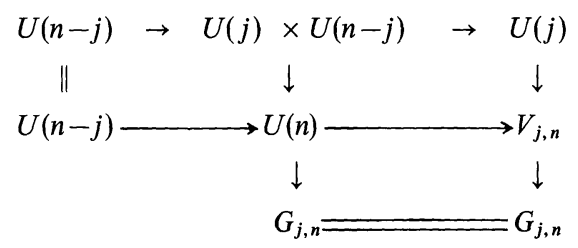

We note that first horizontal diagram is a trivial bundle, and each of the square diagrams commutes.

In Sects. III and IV we showed that the 3-dimensional $\mathbb{C} P^{1}$-model had not a WessZumino-Witten like term due to the topological fact $\pi_{3}\left(\mathbb{C} P^{1}\right)=\mathbb{Z}$. That is, the 3-dimensional $\mathbb{C} P^{1}$-model was our exceptional case. In spite of this fact we can add a Hopf term to the $\mathbb{C} P^{1}$-model by hand. As to this point we refer to

Otsu, H., Sato, T.: "Local" versus "nonlocal" Hopf terms are they equivalent? Mod. Phys. Lett. 17, 1571-1578 (1992)

\section{References}

1. Asada, A.: Introduction to characteristic classes (in Japanese). The Bulletin of Yokohama City University 43, 17-132 (1992)

2. Bowick, M.J., Karabali, D., Wijewardhana, L.C.R.: Fractional spin via canonical quantization of the $O(3)$ nonlinear sigma model. Nucl. Phys. B271, 417-428 (1986)

3. Ferretti, G., Rajeev, S.G.: Current algebra in three dimensions. Phys. Rev. Lett. 69, 2033-2036 (1992)

4. Ferretti, G., Rajeev, S.G.: $\mathbb{C} P^{N-1}$ model with a Chern-Simons term. Preprint (1992)

5. Fujii, K.: A relation between instantons of Grassmann sigma-models and Toda equations: Comments to the paper of DJPT. Preprint (1991), A relation between instantons of Grassmann sigma-models and Toda equations II. Lett. Math. Phys. 25, 203-211 (1992)

6. Fujii, K.: A classical solution of the non-linear complex Grassmann sigma-model with higher derivatives. Commun. Math. Phys. 101, 207-211 (1985)

7. Fujii, K., Tanaka, M.: Universal Schwinger cocycles of current algebra in $(D+1)$-dimensions: Geometry and Physics. Commun. Math. Phys. 129, 267-280 (1990) 
8. Husemoller, D.: Fibre Bundles. New York: McGraw-Hill, 1966

9. Jackiw, R.: Topics in planar physics. In: Physics, Geometry, and Topology. Plenum Press, 1990, pp. 191-239

10. Mickelsson, J.: Current Algebras and Groups. London: Plenum Press, 1989

11. Mickelsson, J., Rajeev, S.G.: Current algebra in $D+1$-dimensions and determinant bundles over infinite dimensional Grassmannians. Commun. Math. Phys. 129, 365-400 (1988)

12. Milnor, J., Stasheff, J.D.: Characteristics Classes. Ann. of Math. Studies 76, Princeton, 1974

13. Pak, N.K.: Canonical structure of the $\mathbb{C} P^{N}$ sigma model in $1+2$ dimensions. Phys. Lett. B260, 377-380 (1991)

14. Steenrod, N.: The Topology of Fibre Bundles. Princeton, NJ: Princeton University Press, 1951

15. Witten, E.: Non-abelian bosonization in two-dimensions. London: Commun. Math. Phys. 92, 455-472 (1984)

16. Zakrzewski, W.J.: Low Dimensional Sigma Models. London: Adam-Hilger, 1989

Communicated by R.H. Dijkgraaf 
